\title{
Non-stoichiometric oxide and metal interfaces and reactions
}

Article

Accepted Version

Bennett, R. A., Mulley, J.S., Basham, M., Nolan, M., Elliott, S.D. and Mulheran, P.A. (2009) Non-stoichiometric oxide and metal interfaces and reactions. Applied Physics A, 96 (3). pp. 543-548. ISSN 0947-8396 doi: https://doi.org/10.1007/s00339008-5066-1 Available at https://centaur.reading.ac.uk/16778/

It is advisable to refer to the publisher's version if you intend to cite from the work. See Guidance on citing.

Published version at: http://dx.doi.org/10.1007/s00339-008-5066-1

To link to this article DOI: http://dx.doi.org/10.1007/s00339-008-5066-1

Publisher: Springer

All outputs in CentAUR are protected by Intellectual Property Rights law, including copyright law. Copyright and IPR is retained by the creators or other copyright holders. Terms and conditions for use of this material are defined in the End User Agreement.

\section{www.reading.ac.uk/centaur}

\section{CentAUR}

Central Archive at the University of Reading

Reading's research outputs online 


\title{
Non-stoichiometric oxide and metal interfaces and reactions
}

R.A. Bennett ${ }^{1}$, J.S. Mulley ${ }^{1}$, M. Basham ${ }^{1}$, M. Nolan ${ }^{2}$, S.D Elliott ${ }^{2}$ and P.A. Mulheran ${ }^{3}$

1 Department of Physics University of Reading, RG6 6AF, UK

2 Tyndall National Institute, Lee Maltings, Prospect Row, Cork, Ireland

3 Department of Chemical and Process Engineering, University of Strathclyde, James Weir Building, 75 Montrose Street, Glasgow G1 1XJ, UK

\begin{abstract}
We have employed a combination of experimental surface science techniques and density functional calculations to study the reduction of $\mathrm{TiO}_{2}(110)$ surfaces through the doping with submonolayer transition metals. We concentrate of the role of $\mathrm{Ti}$ adatoms in self doping of rutile and contrast the behaviour to that of $\mathrm{Cr}$. DFT+U calculations enable identification of probable adsorption structures and their spectroscopic characteristics. Adsorption of both metals leads to a broken symmetry and an asymmetric charge transfer localised around the defect site of a mixed localized/delocalised character. Charge transfer creates defect states with Ti3d character in the bandgap at $\sim 1 \mathrm{eV}$ binding energy. Cr adsorption, however, leads to a very large shift in the valence band edge to higher binding energy and the creation of $\mathrm{Cr} 3 \mathrm{~d}$ states at $2.8 \mathrm{eV}$ binding energy. Low temperature oxidation lifts the $\mathrm{Ti}$ derived bandgap states and modifies the intensity of the $\mathrm{Cr}$ features, indicative of a change of oxidation state from $\mathrm{Cr}^{3+}$ to $\mathrm{Cr}^{4+}$. Higher temperature processing leads to a loss of $\mathrm{Cr}$ from the surface region - indicative of its substitution into the bulk.
\end{abstract}

\section{Introduction}

Reducible transition metal oxides play a key role as active materials in the development of devices such as spintronics, where magnetic properties of the material are harnessed to manipulate spin polarised electron currents; and in gas sensors in which surface chemical reactions induce changes in electronic structure which are picked up by external instruments. In both these examples the subtle interplay between electronic and geometric structure at surfaces and interfaces dictates the material behaviour.

Rutile titanium dioxide $\left(\mathrm{TiO}_{2}\right)$ has become the prototypical reducible system for study due to its relatively simple electronic structure and accessibility in single crystalline form $\left[{ }^{1}\right]$. The material properties are sensitive to the nature of its free surfaces and the presence of oxide-metal interfaces. Non-stoichiometry tends to lead to a semi-conducting bulk with a novel surface chemistry while crystallographic shear plane phases form in the bulk $\left[^{2}\right]$ and give ordered surface structures $\left[{ }^{3}{ }^{7}\right]$ for large departures from stoichiometry, forming a homologous series in the range $\mathrm{Ti}_{\mathrm{n}} \mathrm{O}_{2 \mathrm{n}-1} \mathrm{n}<37$. 
Chromium dioxide $\left(\mathrm{CrO}_{2}\right)$ is also a rutile structured transition metal oxide of importance to the development of spin polarised electronics $\left[{ }^{8}\right]$. It is a half metallic ferromagnet with almost $100 \%$ spin polarisation at the Fermi level such that electron conduction only occurs with one orientation of electron spin, the other spin direction is insulating. The oxide has a Curie temperature of $397 \mathrm{~K}$ so would be widely applicable in electronic devices. However, $\mathrm{CrO}_{2}$ is a metastable oxide and readily decomposes to $\mathrm{Cr}_{2} \mathrm{O}_{3}$ (a more thermodynamically stable oxide) which is an antiferromagnetic insulator. The competition between the formation of these compounds during growth has lead to strategies being developed to support the $\mathrm{CrO}_{2}$ phase and suppress the $\mathrm{Cr}_{2} \mathrm{O}_{3}$, the most successful being epitaxy on a matched support. Rutile $\mathrm{TiO}_{2}$ single crystals are readily available and provide a reasonably good epitaxial match to $\mathrm{CrO}_{2}\left(\mathrm{CrO}_{2}\right.$ is $4.422 \AA \times 2.917 \AA$, $\mathrm{TiO}_{2}$ is $4.584 \AA \times 2.96 \AA$ in the notation $\mathrm{a}=\mathrm{b} \times \mathrm{c}$ axis). It has been shown that $\mathrm{CrO}_{2}$ films can be grown on $\mathrm{TiO}_{2}$ by atmospheric pressure chemical vapour deposition but cannot be successfully grown in clean vacuum conditions, which limits the potential for understanding key properties at a fundamental level. Other approaches to forming spin polarised materials are based on the search for dilute ferromagnetic semiconductors in which dopant atoms are introduced into the oxide. In the case of doped $\mathrm{TiO}_{2}$ host material (usually Co doped anatase $\mathrm{TiO}_{2}$ ) controversy surrounds the nature of the magnetism, particularly in regard of dispersed isolated dopant ions or aggregated clusters of ferromagnetic metal.

In gas sensors the key problems are in obtaining sensitivity and selectivity to the desired gas and in maintaining long operating lifetimes. Recent work on the titanium dioxide surface, a common catalytically active support and sensor material, has shown remarkable complexity in the relationship between surface structure (electronic and geometric) to bulk non-stoichiometry. Furthermore the addition of metal atoms and particles to the surfaces of reducible oxides, forming doped compounds and model heterogeneous catalysts respectively, modifies the surface response to gases.

In this paper we will briefly review our recent findings for metal doped rutile $\mathrm{TiO}_{2}$ single crystal surfaces and in particular highlight new work in comparing the behaviour of self-doped nonstoichiometric $\mathrm{TiO}_{\mathrm{x}}$ and $\mathrm{Cr}$ doped $\mathrm{TiO}_{2}$. The importance here is to identify the structure and charge transfer that occurs at the interface as this controls the local electronic structure of relevance to growth, sensing and magnetism. We draw heavily on detailed first principles calculations of these systems to gather insights of relevance to gas sensors and the epitaxial growth of oxides, in particular attempts to grow $\mathrm{CrO}_{2}$ on $\mathrm{TiO}_{2}$.

\section{Methods}

The experiments were carried out in a twin chamber UHV system described previously [ $\left.{ }^{9}\right]$ with the preparation chamber equipped with a Vacuum Generators low energy electron diffraction 
(LEED) system, Oxford Applied Research water-cooled e-beam evaporator for $\mathrm{Cr}$ and Ti deposition and additional facilities for $\mathrm{Ar}^{+}$or $\mathrm{O}^{+}$ion sputtering. Photoelectron spectroscopy was undertaken in a second mu-metal chamber and equipped with a hemispherical electron energy analyser, which can be used in conjunction with an $\mathrm{Al} \mathrm{K \alpha}$ X-ray source to perform X-ray photoelectron spectroscopy (XPS), or with a Helium lamp to perform ultra-violet photoelectron spectroscopy (UPS). A gate valve separates the two chambers so that the analyser is never exposed to large reactive gas loads that might change its workfunction. Photoelectron spectra were analysed with CASAXPS with Shirley backgrounds and decomposed into Gaussian Lorentzian mixed product curves for quantification. Stoichiometry of the $\mathrm{TiO}_{2}$ crystal is defined by the ratio (expressed as a percentage) of $\mathrm{Ti}^{4+}$ states: all $\mathrm{Ti}$ states identified by core-level shifts in high resolution XPS. This definition avoids complications with sensitivity factors in comparing $\mathrm{Ti}$ and $\mathrm{O} 1 \mathrm{~s}$ peaks. Deposited fluxes are calculated from calibration experiments by growth of pseudomorphic monolayers on W(100) single crystals $\left[{ }^{10}\right]$. $\mathrm{TiO}_{2}(110)$ single crystal samples (PI-KEM, UK) were cleaned by $\mathrm{O}^{+}$ion sputtering and low temperature $\left(\mathrm{O}_{2} 1 \times 10^{-7}\right.$ mbar $)$ anneal cycles to prevent the formation of bulk defects and became only lightly coloured during experiments. The samples thus prepared were very close to stoichiometric as indicated by XPS.

All calculations are carried out in the framework of the periodic plane wave approach using density functional theory (DFT) with the VASP code $\left[{ }^{11}\right]$. In this approach, the valence electronic states are expanded in a basis of periodic plane waves, with a converged plane wave energy cut-off of $396 \mathrm{eV}$ from $\left[{ }^{12}\right]$. This plane wave cut off energy is consistent with many studies of metal oxides. The core-valence interaction is treated using PAW potentials $\left[{ }^{13}\right]$. The PW91 exchange-correlation functional $\left[{ }^{14}\right]$ is used. The surface is studied as a finite thickness slab model with three-dimensional periodicity; each slab is separated using a vacuum thickness of $10 \AA$ along the z-direction (perpendicular to the surface). The slab model has 6 repeat O-Ti-O units (giving 18 atomic layers) and a $(2 \times 4)$ expansion of the surface cell. For the adsorbate- $\mathrm{TiO}_{2}$ interaction, this surface cell expansion minimises periodic adsorbate-adsorbate interactions, provides a concentration of adsorbed atoms that is consistent with experimentally realisable concentrations and is a realistically large system ( 290 atoms). The Ti/Cr atom is adsorbed on one side of the slab and a dipole correction is added to remove the resulting dipole perpendicular to the surface. The $\mathrm{Cr}$ atom has a 4s1 3d5 configuration, and we carry out spin polarised calculations, with the spin degrees of freedom allowed to relax. The large size of cell expansion, and the fact that the oxide is insulating, allows k-point sampling to be performed at the $\Gamma$-point.

A novel aspect of these calculations concerns the theoretical description of the electronic structure of the transition metal ions $\mathrm{Cr}$ and Ti. Reduced transition metal ions such as $\mathrm{Ti}$ and $\mathrm{Cr}$, which have partially occupied $3 \mathrm{~d}$ shells $\left[{ }^{15-21}\right]$, are difficult to describe due to the incomplete 
cancellation of the self-interaction error in approximate DFT exchange-correlation functionals. Thus, we use DFT corrected for on-site Coulomb interactions, (DFT+U), in all calculations of the adsorbate structures. $\mathrm{U}$ is set to $3 \mathrm{eV}$ for $\mathrm{Ti}$, consistent with previous computations $[14,16]$ and for the $\mathrm{Cr}$ adsorbate, since the partially filled $\mathrm{Cr} 3 \mathrm{~d}$ shell also cannot be described with DFT [17-20].

\section{Ti on $\mathrm{TiO}_{2}(110)$}

Five possible reactions for the adsorption of a single $\mathrm{Ti}$ atom on the (110) surface can be considered $\left[{ }^{22}\right]$. Using simple chemical equations, these are:

1. $\mathrm{Ti}^{0}+\mathrm{Ti}^{4+} \mathrm{O}^{2-} \mathrm{O}^{2-} \rightarrow \mathrm{Ti}^{2+}{ }_{2} \mathrm{O}^{2-} \mathrm{O}^{2-}$

2. $\mathrm{Ti}^{0}+3\left(\mathrm{Ti}^{4+} \mathrm{O}^{2-} \mathrm{O}^{2-}\right) \rightarrow \mathrm{Ti}^{3+}+3\left(\mathrm{Ti}^{3+} \mathrm{O}^{2-} \mathrm{O}^{2-}\right)$

3. $\mathrm{Ti}^{0}+2\left(\mathrm{Ti}^{4+} \mathrm{O}^{2-} \mathrm{O}^{2-}\right) \rightarrow \mathrm{Ti}^{2+}+2\left(\mathrm{Ti}^{3+} \mathrm{O}^{2-} \mathrm{O}^{2-}\right)$

4. $\mathrm{Ti}^{0}+\mathrm{Ti}^{4+} \mathrm{O}^{2-} \mathrm{O}^{2-} \rightarrow \mathrm{Ti}^{0}+\mathrm{Ti}^{4} \mathrm{O}^{2-} \mathrm{O}^{2-}$

5. $\mathrm{Ti}^{0}+\left(\mathrm{Ti}^{4+} \mathrm{O}^{2-} \mathrm{O}^{2-}\right)_{\mathrm{n}} \rightarrow \mathrm{Ti}^{\mathrm{m}+}+\left(\mathrm{Ti}^{4} \mathrm{O}^{2-} \mathrm{O}^{2-}\right)_{\mathrm{n}}{ }^{\mathrm{m}-}$

where $\mathrm{Ti}^{0}$ is the neutral $\mathrm{Ti}$ atom that adsorbs to the surface and $\mathrm{Ti}^{4+} \mathrm{O}^{2-} \mathrm{O}^{2}$ is a surface $\mathrm{TiO}_{2}$ unit. The final state of the adsorbate-surface differs in each scheme depending on the detailed interactions: (1) a Ti atom interacting with a single surface Ti ion; (2) a Ti atom interacting with $3 \mathrm{Ti}$ ions; (3) a Ti atom interacting with two surface Ti ions; (4) there is no charge transfer between the Ti atom and the surface (and is not further considered) and (5) where any charge transfer is delocalised over the whole of the system.

Figure 1 shows in the lower spectra Ti $2 p$ XPS and UPS results for single crystal $(1 \times 1)$ $\mathrm{TiO}_{2}(110)$ surface with high stoichiometry $\sim 91 \%$, which is typical of UHV prepared surface showing a good $(1 \times 1)$ LEED pattern. Three oxygen valence peaks at 4.31, 5.81 and 7.78eV binding energy (zero being aligned at the Fermi level) are apparent in the UPS spectra. There is also some weak intensity in the band gap region originating from He satellite lines at 23.09, 23.75 and $24.05 \mathrm{eV}$ which have low intensities in the unmonochromated source $\left[{ }^{23}\right]$. The valence band edge is located at $2.76 \mathrm{eV}$. The results of deposition of $1 / 8 \mathrm{ML} \mathrm{Ti}$ are shown in the upper spectra in which the Ti2p peaks develop a chemical core level shifted component (an apparent stoichiometry of 79\%) and a new band gap state appears at $\sim 1.0 \mathrm{eV}$ below $\mathrm{E}_{\mathrm{F}}$ in UPS. This defect state is asymmetric and clearly separated from the Fermi level. The valence band edge shifts to $3.08 \mathrm{eV}$ and the O2p peaks adopt a different appearance due to shifts in intensity and broadening. The movement of the valence band edge upon adsorption of $\mathrm{Ti}$ at the surface is expected as Ti donates electrons to the bulk setting up a dipole, which bends the bands at the surface. 

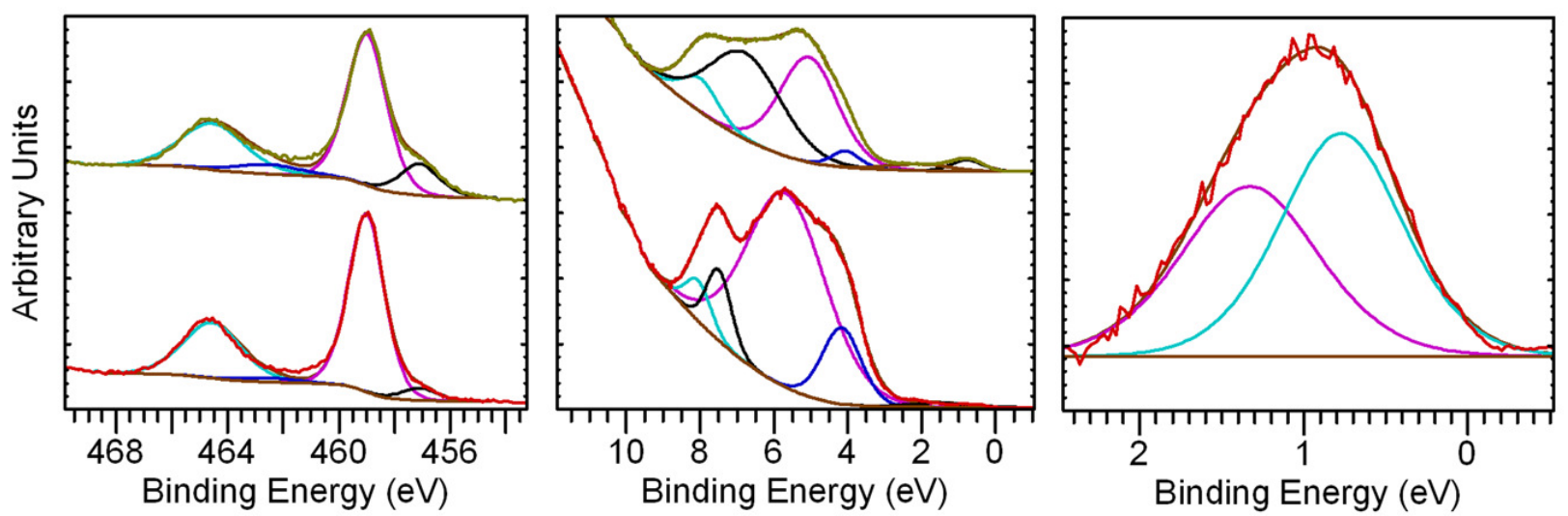

Figure 1. Left panel shows XPS spectra for the near stoichiometric $(1 \times 1)$ surface (lower trace) and $1 / 8 \mathrm{ML}$ Ti deposited surface (upper trace). The spectra have been aligned with $\mathrm{Ti}^{4+}$ at $459 \mathrm{eV}$ and representative peaks fitted to indicate the derivation of stoichiometry. The centre panel shows the same surfaces in UPS with the dramatic changes in the lowest binding energy $\mathrm{O} 2 \mathrm{p}$ state and creation of a defect state upon $\mathrm{Ti}$ adsorption. The rightmost panel shows a difference spectra between stoichiometric and doped surfaces in the bandgap.

The DFT+U calculations predict that the lowest energy structure is an adsorbed atom in a three-fold $\mathrm{O}$ - coordinated site and this structure is shown in Figure 2. The DFT+U predicts the distance from the adsorbed Ti to the nearest 6-fold coordinated Ti to be $2.56 \AA$. For the 5-fold Ti ions nearest the adsorbed atom, the Ti- $\mathrm{O}^{\mathrm{ip}}$ distances fall between $2.008-2.125 \AA$, compared to $1.96 \AA$ in the stoichiometric surface. For the other 5-fold Ti atoms in the same row along (1 $\overline{1} 0)$, elongated Ti-O ${ }^{\text {ip }}$ distances are observed. There is a strong asymmetry in the surface Ti-O distances around the adsorption site with the 6-fold $\mathrm{Ti}$ atom nearest the adsorbed atom is displaced from its lattice site, causing a lengthening of the $\mathrm{Ti}-\mathrm{O}^{\mathrm{br}}$ distances involving this atom.

The excess spin density is introduced by adsorption of Ti. The spin density isosurfaces (the difference between the majority and minority spin electron density) permits analysis of the location of the excess charge and is also shown in Figure 2. One surface 5-fold Ti atom neighboring the adsorption site is reduced to $\mathrm{Ti}^{3+}$, while another 5 -fold surface $\mathrm{Ti}$ atom is partially reduced. The remaining charge is delocalized over surface layer Ti atoms constrained close to the adsorption site, each with a very small degree of reduction and the adsorbed $\mathrm{Ti}$ is in the $2+$ oxidation state. Thus, there is a coupling between localisation of charge on a 5-fold coordinated $\mathrm{Ti}$ atom and a distortion of the atomic structure around that atom, similar to a Jahn-Teller effect; occupation of a Ti 3dorbital with one electron causes a breaking of orbital degeneracy and this is achieved by a structural distortion, yielding significantly elongated Ti-O distances. 


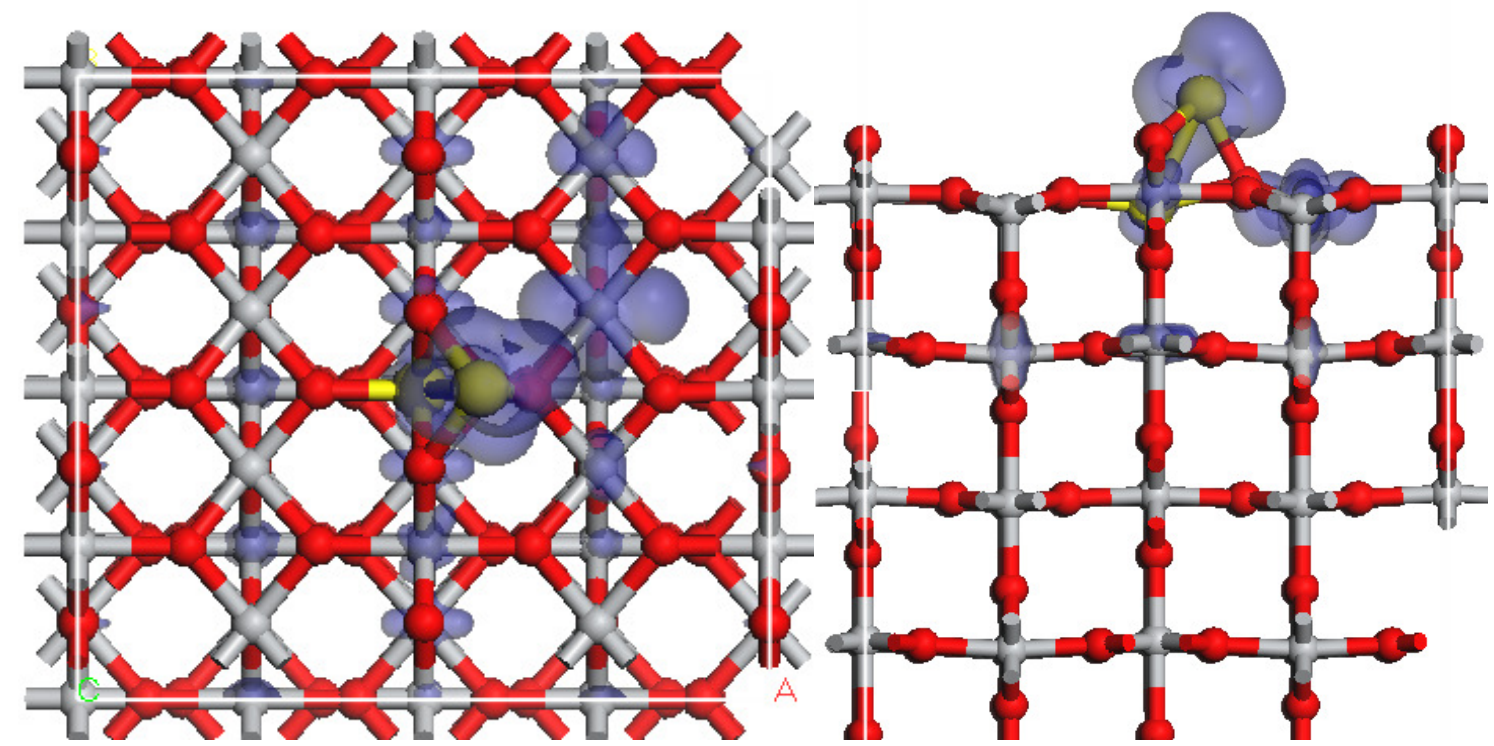

Figure 2: Adsorption structure for $\mathrm{Ti}$ at the (110) $\mathrm{TiO}_{2}$ surface with plan and side view.

\section{$\mathrm{Cr}$ on $\mathrm{TiO}_{2}(110)$}

Cr-doped single crystal $(1 \times 1) \mathrm{TiO}_{2}(110)$ experiments were performed by starting with the clean surface, doping with $1 / 8 \mathrm{ML}$ Cr adatoms, subsequently oxidising the surface in $1 \times 10^{-7}$ torr oxygen for $5 \mathrm{~min}$ at $318 \mathrm{~K}$, and at $573 \mathrm{~K}$. Figure 3 shows UPS spectra obtained for these four surfaces. Upon doping the surface with $\mathrm{Cr}$ two defect states appear in the bandgap with binding energies of $\sim 2.8 \mathrm{eV}$ and $\sim 1.0 \mathrm{eV}$, which are $\mathrm{Cr} 3 d$ and $\mathrm{Ti} 3 d$ derived respectively. The valence band edge is shifted to higher binding energy from $3.0 \mathrm{eV}$ to $3.7 \mathrm{eV}$ and the valence states exhibit a similar broadening and shift to lower intensity when compared to the self (Ti) doped surface. After performing the lower temperature oxidation the $\mathrm{Ti}$ defect state is no longer observed and the $\mathrm{Cr}$ defect state shifts to a lower binding energy $(\sim 2.4 \mathrm{eV})$ and the valence band edge is shifted back to $3.0 \mathrm{eV}$. The intensity of the $\mathrm{Cr}$ state (referenced to the valence band emission) is reduced to $2 / 3$ of the intensity of the as doped surface, which immediately suggests a change of oxidation state from $\mathrm{d}^{3} \mathrm{Cr}^{3+}$ to $\mathrm{d}^{2} \mathrm{Cr}^{4+}$. Upon high temperature oxidation the $\mathrm{Cr}$ defect vanishes (due to no $\mathrm{Cr}$ in the surface region - see XPS data) but more notably the Ti $3 d$ state is reintroduced at $0.9 \mathrm{eV}$. 

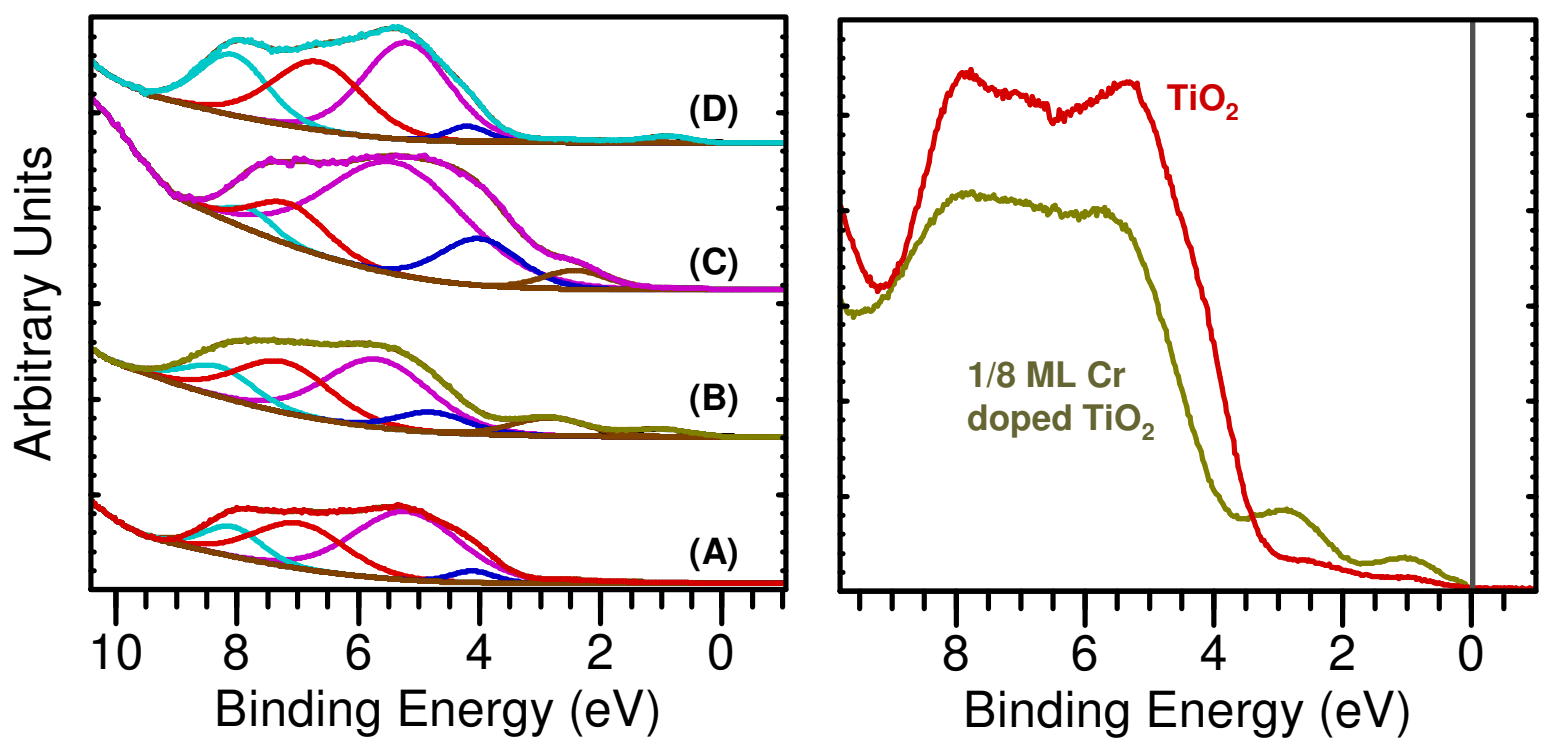

Figure 3. Left panel displays He I UPS spectra for (A) clean $\mathrm{TiO}_{2}(110)$, (B) 1/8 ML Cr-doped TiO 2 (110), (C) 1/8 ML Cr-doped $\mathrm{TiO}_{2}(110)$ oxidised at $318 \mathrm{~K}$ and (D) $1 / 8 \mathrm{ML}$ Cr-doped $\mathrm{TiO}_{2}(110)$ oxidised at $573 \mathrm{~K}$. Right Panel overlays the spectra from (A) and (B) and is normalised at the Fermi level.

Figure 4 displays XPS spectra taken for the four surfaces. The Ti $2 p$ spectra reveals the stoichiometry of the clean $\mathrm{TiO}_{2}(110)$ as $90.4 \%$. Doping the surface with $1 / 8 \mathrm{ML}$ Cr reduces the stoichiometry to $81.2 \%$. Of major significance, however, is the large increase in stoichiometry to 95.3\% which occurs during the low temperature oxidation. Here the low temperature Cr oxidised surface exhibits a $~ 5 \%$ larger stoichiometry than the clean well prepared surface suggesting a strong electronic effect. Once the surface is oxidised at $573 \mathrm{~K}$ the stoichiometry is reduced to a value commensurate with the clean starting substrate. Analysis of the $\mathrm{Cr} 2 \mathrm{p}$ peak data is complicated by the Ti 2 s state $(\sim 565 \mathrm{eV})$ and its associated shake-up and shake-off satellites, which extend through the $\mathrm{Cr} 2 \mathrm{p}$ region $(570-590 \mathrm{eV})$. In order to extract the $\mathrm{Cr} 2 \mathrm{p}_{3 / 2}$ and $\mathrm{Cr} 2 \mathrm{p}_{1 / 2}$ peak data clean $\mathrm{TiO}_{2}(110)$ reference spectra was recorded and fitted as a background to the Cr-doped spectra. From figure 4 it is seen that no $\mathrm{Cr}$ peaks are seen for the clean surface (A) nor for the high temperature (573K) oxidation (D) which suggests either the $\mathrm{Cr}$ has either been desorbed from the surface or $\mathrm{Cr}$ has been absorbed into the bulk and the signal from the surface sufficiently low so that the $\mathrm{Cr} 2 \mathrm{p}$ signal is buried within the Ti 2 s signal. Doping the sample with $\mathrm{Cr}$ introduces two clear peaks at $575.7 \mathrm{eV}$ and $586.0 \mathrm{eV}$ attributed to the oxidised $\mathrm{Cr} 2 \mathrm{p}_{3 / 2}$ and $\mathrm{Cr} 2 \mathrm{p}_{1 / 2}$ states respectively $\left(\mathrm{Cr}^{0}\right.$ and $\mathrm{Cr}^{3+}$ are reported at $574.1 \mathrm{eV}\left[{ }^{24}\right]$ and $\left.562.2-577.3 \mathrm{eV}\left[{ }^{25}\right]\right)$. After oxidation at $318 \mathrm{~K}$ the $\mathrm{Cr} 2 \mathrm{p}_{3 / 2}$ is shifted to $577.2 \mathrm{eV}$. For all four spectra no discernable shift is observed in the $\mathrm{O} 1 \mathrm{~s}$ state, which is centred on $530.3 \mathrm{eV}$. 

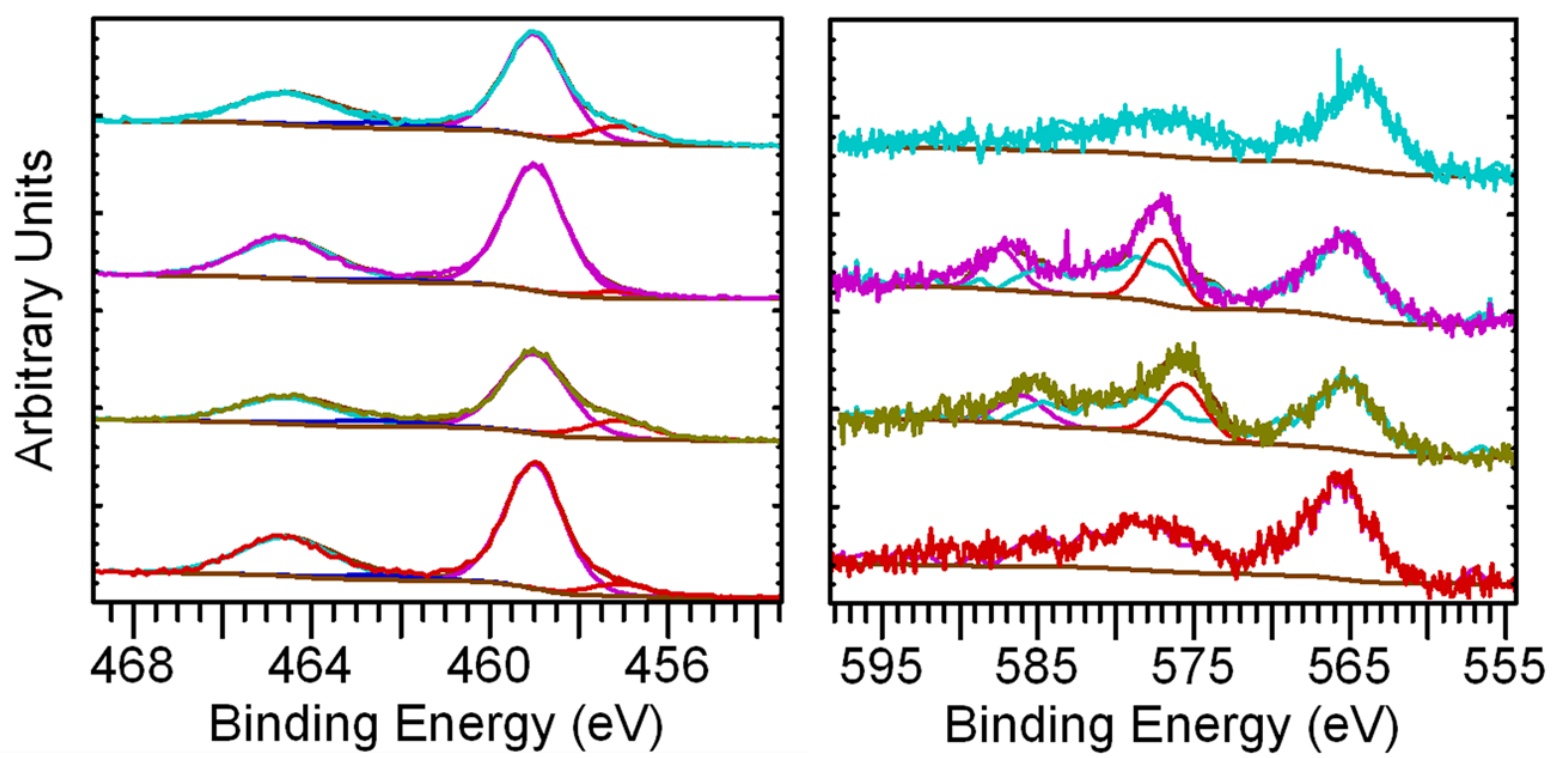

Figure 4. XPS spectra obtained for (A) clean $\mathrm{TiO}_{2}(110)$, (B) $1 / 8 \mathrm{ML}$ Cr-doped $\mathrm{TiO}_{2}(110)$, (C) $1 / 8 \mathrm{ML}$ Cr-doped $\mathrm{TiO}_{2}(110)$ oxidised at $318 \mathrm{~K}$ and (D) $1 / 8 \mathrm{ML} \mathrm{Cr}$-doped $\mathrm{TiO}_{2}(110)$ oxidised at $573 \mathrm{~K}$. The left panel displays the Ti $2 \mathrm{p}$ region and the right panel the $\mathrm{Cr} 2 \mathrm{p} / \mathrm{Ti} 2$ s region.

The peak shift of $1.5 \mathrm{eV}$ between the as-doped surface and the low temperature oxidised surface indicates either a change in oxidation state or a significant band bending at the surface. In combination with the valence band shifts and changes in peak areas in the UPS experiments suggests both effects happening in concert. The difference between $2 \mathrm{p}_{3 / 2} \mathrm{Cr}^{3+}$ and $\mathrm{Cr}^{4+}$ binding energies is small $\left(\mathrm{Cr}^{4+}\right.$ in $\mathrm{CrO}_{2}$ is $0.5 \mathrm{eV}$ lower in binding energy that $\mathrm{Cr}^{3+}$ in $\left.\mathrm{Cr}_{2} \mathrm{O}_{3}\left[{ }^{26}\right]\right)$ and may well be masked.

The DFT+U results predict that the most stable adsorption structure for $1 / 16 \mathrm{ML} \mathrm{Cr}$ on $\mathrm{TiO}_{2}$ has the $\mathrm{Cr}$ atom bridging two two-fold coordinated bridging oxygen atoms, figure 5(a). The $\mathrm{Cr}$ - bridging $\mathrm{O}$ distances are $1.95 \AA$ while the distance from $\mathrm{Cr}$ to the 6-fold coordinated surface $\mathrm{Ti}$ atom (Ti ${ }^{6 \text { fold }}$ ) below the adsorbate is $2.83 \AA$ and $\mathrm{Cr}$ lies $2.44 \AA$ above the surface plane. The most important feature of the geometry is the strong asymmetry in the distances involving the 5-fold coordinated surface $\mathrm{Ti}$ atoms along the rows either side of the adsorbate. In the row immediately left of the adsorbate the $\mathrm{Ti}^{5 \text {-fold }}-\mathrm{O}^{\text {in-plane }}$ distances range from $1.92-2.05 \AA$, with the longest distances (the most distorted geometry) centred around one 5-fold $\mathrm{Ti}$ atom. However in the other row, the corresponding $\mathrm{Ti}^{5 \text {-fold }}-\mathrm{O}^{\text {in-plane }}$ distances are little distorted from the stoichiometric surface, ranging from $1.97-1.98 \AA$. Therefore upon adsorption of $\mathrm{Cr}$, there is a structural distortion and creation of asymmetry as was observed for $\mathrm{Ti}$ doping of $\mathrm{TiO}_{2}$. Similarly to $\mathrm{Ti}$ adsorption, there is a mixture of localised and delocalised charge, figure 5(a), with a 5-fold coordinated Ti atom showing charge localisation and this is the Ti atom showing the elongated Ti-O distances discussed above. Finally, the DOS in figure 5(b), shows that $\mathrm{Cr}$ adsorption also produces a state in the $\mathrm{TiO}_{2}$ band gap, with 
the $\mathrm{Cr}$ derived state lying closer the valence band than the $\mathrm{Ti}$ derived state. The $\mathrm{Cr}$ atom is oxidised to $\mathrm{Cr}(\mathrm{II})$, with 4 unpaired d electrons.
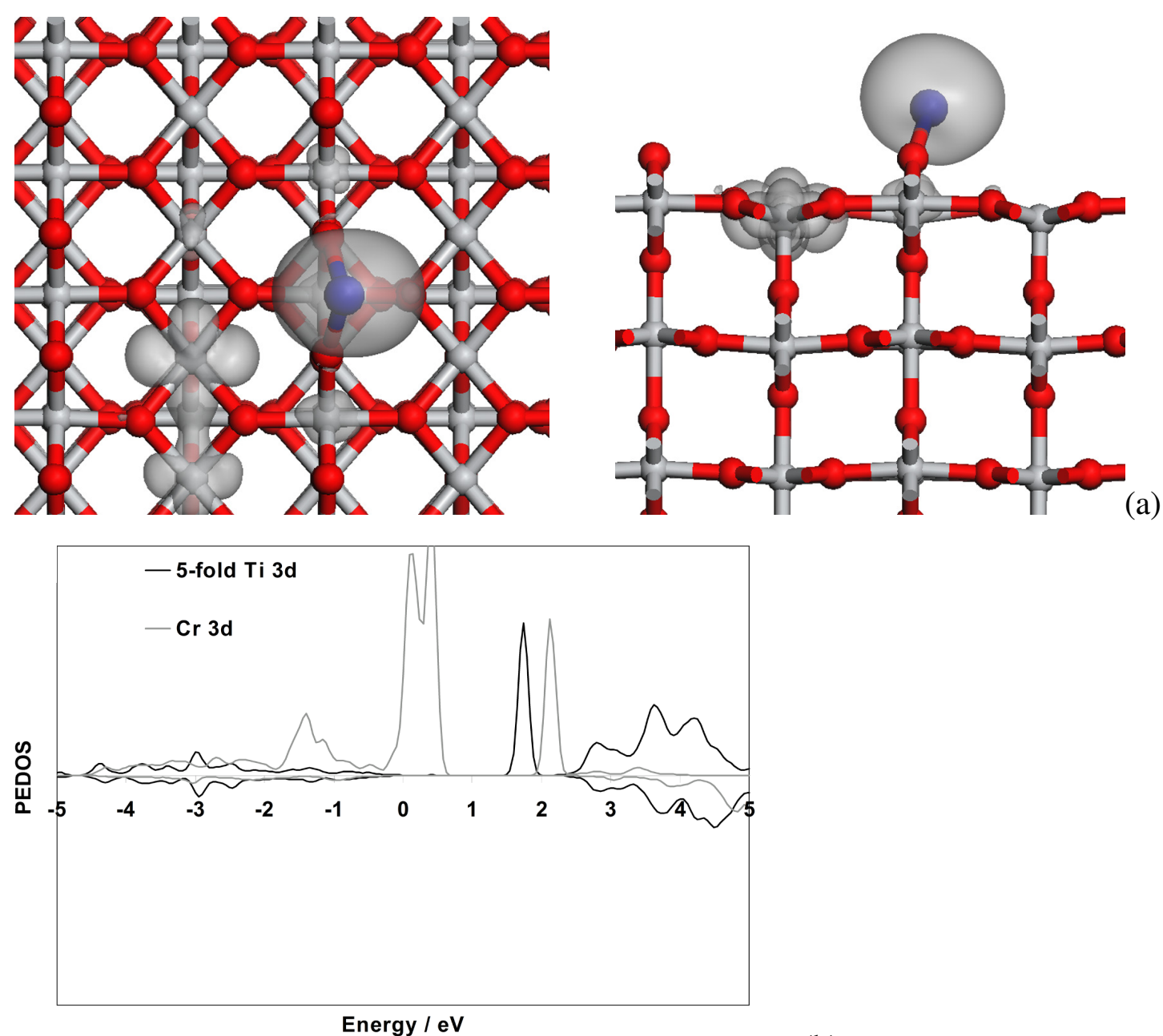

(b)

Figure 5: (a): Adsorption structure of $\mathrm{Cr}$ on the (110) surface of $\mathrm{TiO}_{2}$ at $1 / 16 \mathrm{ML}$ coverage with excess spin density indicated. (b): Density of states projected on $\mathrm{Cr}$ and Ti $3 \mathrm{~d}$ states

\section{Conclusions}

The adsorption of $\mathrm{Ti}$ and $\mathrm{Cr}$ on the surface of $\mathrm{TiO}_{2}$ induces charge transfer and band bending that changes the surface electronic structure. In both cases the adsorption leads to a broken symmetry and an asymmetric charge transfer which is localised around the defect site and leads to a mixed localized/delocalised character. The experimental results correlate well with the ab-initio modelling and show that both $\mathrm{Cr}$ and $\mathrm{Ti}$ are partially oxidised upon adsorption resulting in the formation of distinct band gap states. The charge transfer and reactivity of the doped surfaces was investigated by oxygen adsorption and thermal annealing which indicated that $\mathrm{Cr}$ was easily substituted into the lattice at high temperatures but gave an unusually strong band bending with low temperature adsorption.

\section{Acknowledgments}


The Royal Society/Wolfson foundation is gratefully acknowledged for a refurbishment grant for laboratories in Reading and JSM would like to thank the University of Reading Research Endowment Trust Fund for a studentship. PAM would like to thank the EPSRC for project GR/T18738/01. We acknowledge the European Commission for support (NATCO), the Science Foundation Ireland funded computational resources at Tyndall and the SFI/HEA funded Irish Centre for High Performance Computing (ICHEC) for provision of computing resources.

\section{References}

${ }^{1}$ U. Diebold, Surf. Sci. Rep., 2003, 48, 53,.

${ }^{2}$ L. A. Bursill and B. G. Hyde, in Progress in Solid State Chemistry, ed. H. Reiss and J. O. McCaldin 7, p. 177 (Pergamon Press, New York, 1972).

${ }^{3}$ G.S. Rohrer, V.E. Henrich and D.A. Bonnell, Science, 1990, 250, 1239

${ }^{4}$ G.S. Rohrer, V.E. Henrich and D.A. Bonnell, Surf. Sci. 1992, 278, 146

${ }^{5}$ R.A. Bennett, S. Poulston, P. Stone and M. Bowker, Phys. Rev. B 1999, 59, 10341

${ }^{6}$ H. Nörenberg, R.E. Tanner, K.D. Schierbaum, S. Fischer and G.A.D. Briggs, Surf. Sci., 1998, 396, 52

${ }^{7}$ H. Nörenberg and G.A.D. Briggs, Surf. Sci., 1998, 402-404, 738-741

${ }^{8}$ J.M.D. Coey and M. Venkatesan, J. Appl. Phys., 2002, 91, 8345.

${ }^{9}$ R.A. Bennett, J.S. Mulley, M.A. Newton and M. Surman, J. Chem. Phys., 2007, 127, 084707

${ }^{10}$ N.D. McCavish and R.A. Bennett, Surf. Sci., 2003, 546, 47

${ }^{11}$ G. Kresse, and J. Hafner, Phys. Rev. B, 1994, 49, 14251; G. Kresse and J. Furthmüller, Comp. Mat. Sci. 1996, 6, 15

${ }^{12}$ S. D. Elliott and S. P. Bates, Phys. Rev. B, 2002, 65, 245415

${ }^{13}$ P. E. Blöchl, Phys. Rev. B 1994, 50, 17953; D. Joubert and G. Kresse, Phys. Rev. B 1999, 59, 1758

${ }^{14}$ J. P. Perdew, in: Ziesche, P.; Eschrig H. (Eds.), Electronic Structure of Solids '91, Akademie Verlag, Berlin, 1991

${ }^{15}$ D. D. Cuong, B. Lee, K. M. Choi, H. S. Ahn, S. Han, S. and J. Lee, Phys. Rev. Lett., 2007, 98, 115503

${ }^{16}$ R. Pentcheva, and W. E. Pickett, Phys. Rev. B, 2006, 74, 035112

${ }^{17}$ A. I. Poteryaev, A. I. Lichtenstein, and G. Kotliar, Phys. Rev. Lett., 2004, 93, 086401

${ }^{18}$ A. Rohrbach, J. Hafner and G. Kresse, Phys. Rev. B, 2004, 70, 125426

${ }^{19}$ L. Wang, T. Maxisch and G. Ceder, Phys. Rev. B, 2006, 73, 195107

${ }^{20}$ D. L. Niu and X. J. Liu, J. Luminesence, 2007, 122-123, 155

${ }^{21}$ M. Korotin, V. I. Anisimov, D. I. Komskii and G. A. Sawatzky, Phys. Rev. B, 1998, 80, 4305

${ }^{22}$ N. D. McCavish and R. A. Bennett, Topics in Catalysis, 2005, 36, 11

${ }^{23}$ M. Cardona and L. Ley eds. "Photoemission in solids I", Topics in Applied Physics Vol 26, Springer-Verlag, 1978.

${ }^{24}$ C.P. Huggins and R.M. Nix, Surf. Sci., 2005, 594, 163

${ }^{25}$ V. Maurice, S.Cadot and P. Marcus, Surf. Sci., 2000, 458, 195.

${ }^{26}$ R. Cheng, B. Xu, C.N. Borca, A. Sokolov, C.-S. Yang, L. Yuan, S.-H. Liou, B. Doudin and P.A. Dowben, Appl. Phys. Lett., 2001, 79, 3122. 